\title{
Combination immunotherapy in metastatic urothelial cancer: time for the next step?
}

\author{
Panagiota Economopoulou, Aristotelis Bamias
}

Second Propaedeutic Department of Internal Medicine, Medical School, National \& Kapodistrian University of Athens, Attikon University Hospital, Athens, Greece

Correspondence to: Prof. Aristotelis Bamias. Second Propaedeutic Department of Internal Medicine, Medical School, National \& Kapodistrian University of Athens, Attikon University Hospital, 1st Rimini St., 12462 Haidari, Athens, Greece. Email: abamias@med.uoa.gr.

Provenance and Peer Review: This article was commissioned by the Editorial Office, Chinese Clinical Oncology. The article did not undergo external peer review. Comment on: Sharma P, Siefker-Radtke A, de Braud F, et al. Nivolumab Alone and With Ipilimumab in Previously Treated Metastatic Urothelial Carcinoma: CheckMate 032 Nivolumab $1 \mathrm{mg} / \mathrm{kg}$ Plus Ipilimumab 3 mg/kg Expansion Cohort Results. J Clin Oncol 2019;37:1608-16.

Submitted Oct 25, 2019. Accepted for publication Oct 31, 2019.

doi: $10.21037 /$ cco.2019.10.07

View this article at: http://dx.doi.org/10.21037/cco.2019.10.07

Over the past 40 years, cisplatin-based chemotherapy has remained the standard of care for metastatic urothelial carcinoma (mUC) (1). However, inadequate renal function and poor performance status preclude the use of platinum-based regimens in approximately $50 \%$ of patients with mUC (2). Moreover, until recently, second line chemotherapy has provided modest benefit in patients who experience disease progression following first line treatment. Nevertheless, since 2016, the approval of immune checkpoint inhibitors (ICIs) for the treatment of mUC has marked a therapeutic renaissance for systemic treatment of $\mathrm{mUC}$. Indeed, five agents have received FDA approval for platinum-refractory disease; among them, pembrolizumab and atezolizumab have been also approved for cisplatin-unfit patients in the frontline setting (3-8).

Clinical efficacy of immunotherapy in $\mathrm{mUC}$ has been shown in numerous clinical trials, but response rates remain relatively low. On the other hand, clinical data in other solid tumors have shown that combining immunotherapies with different targets can provide more effective blockade, maximize efficacy and improve patient outcomes. In this context, the results of the Checkmate 032 study were recently reported (9). Checkmate 032 is an open-label, multicohort study that included patients with platinum-resistant $\mathrm{mUC}$ treated with nivolumab $3 \mathrm{mg} / \mathrm{kg}$ monotherapy every 2 weeks (NIVO3), nivolumab $3 \mathrm{mg} / \mathrm{kg}$ plus ipilimumab $1 \mathrm{mg} / \mathrm{kg}$ every 3 weeks for four doses followed by nivolumab monotherapy $3 \mathrm{mg} / \mathrm{kg}$ every 2 weeks (NIVO3 + IPI1), or nivolumab $1 \mathrm{mg} / \mathrm{kg}$ plus ipilimumab
$3 \mathrm{mg} / \mathrm{kg}$ every 3 weeks for four doses followed by nivolumab monotherapy $3 \mathrm{mg} / \mathrm{kg}$ every 2 weeks (NIVO1 + IPI3).

Checkmate 032 has provided the first indication that combined programmed cell death (PD-1) and cytotoxicT-lymphocyte antigen-4 (CTLA-4) inhibition could have clinical utility in the treatment of mUC. Indeed, the combination NIVO1/IPI3 resulted in higher overall response rate (ORR) and longer overall survival (OS) as compared to NIVO3 and NIVO3/IPI1 arms. Interestingly, NIVO1/IPI3 generated a particularly high ORR (38\% per investigator), which is the highest ever reported for platinum pretreated disease in mUC. More importantly, median OS with NIVO1/IPI3 was shown to be 15.3 months, which is very promising, given that checkpoint inhibitor monotherapy in previously treated $\mathrm{mUC}$ has traditionally provided a median OS of 6.5 to 10.5 months (10). In spite of these very important results, several aspects of this study are worth discussing.

The design of the study does not allow direct comparison of treatment arms, since each have a different length of follow-up. Therefore, this is a question generating effort. The imbalance of the three arms for the most important prognostic factor in this setting, namely the Bellmunt risk stratification (11) strongly underlines this limitation. Nevertheless, since the imbalance was in favor of the nivolumab monotherapy cohort, it could be speculated that the efficacy of NIVO/IPI combinations was underestimated. A scientifically sound comparison of the three arms becomes 
more important, when the increased toxicity observed with the combinations is taken into consideration. Grade 3 or 4 events occurred in approximately $40 \%$ of patients receiving NIVO/IPI. This is in concert with experience from other solid tumors. In melanoma, an even higher percentage of patients $(59 \%)$ receiving this combination experienced grade 3-4 events (12) and although the combination is FDA approved for the disease in the first line setting, high toxicity rate hampers wide use of this regimen.

Why this combination seems more effective than PD-1 inhibition monotherapy? Treatment with PD-1 or PDL1 inhibitors alone produces long lasting responses only in about $25 \%$ of patients (13). This could be, at least partially, attributed to the complexity of anti-tumor immune response. PD-1/PD-L1 blockade affects only the last step of this process (14). Combination of ICIs with different therapeutic targets could, therefore, be more effective. CTLA- 4 hampers early activation and differentiation of $\mathrm{T}$ cells and thus affects a different step of immune response (15). Consequently, their combination can generate complementary effects on effector T cells. This notion is supported by the efficacy of this combination in other tumor types. Nivolumab and ipilimumab combination has now become an acceptable therapeutic option in treatment naïve metastatic melanoma and a standard of care in intermediate and high-risk renal cell carcinoma, based on landmark phase III clinical trials $(12,16)$. In melanoma, the combination of nivolumab and ipilimumab at the same doses used in Checkmate 032 produced an ORR of $58 \%$ and an OS rate at 3 years equal to $58 \%$ (17). In renal cell carcinoma, the combination of nivolumab at a dose of $3 \mathrm{mg} / \mathrm{kg}$ and ipilimumab at a dose of $1 \mathrm{mg} / \mathrm{kg}$ demonstrated an ORR of $42 \%$ and an 18 -month OS rate of $75 \%$ (16). In lung cancer, clinical efficacy of the combination has been also promising $(18,19)$.

The results of this study should be viewed in a rapidly changing treatment paradigm in mUC. Will nivolumab and ipilimumab become the standard of care in the near future? In spite of the limitations of the comparison reported here, the NIVO1/IPI3 combination has been used as the experimental, immunotherapy-pure, arm in the ongoing phase III Checkmate 901 trial, which compares this combination with the standard of care in the first line setting. This study, as well as others, also included PD-1 or PD-L1 inhibition in combination with chemotherapy. If these ongoing studies are positive, it seems likely, that after forty years of anticipation and continuous efforts, the treatment landscape in mUC may dramatically change due to the endorsement of immunotherapy-containing combinations in the first line setting.

Selection of patients is a fundamental element of targeted therapy, which includes immunotherapy. Clinical factors are already used in other tumors, such as renal cancer, within this context (16). In bladder cancer, however, molecular markers have shown promise. In the study of Sharma et al., patients with high PD-L1 expression appeared to live longer, regardless of treatment received (9). Particularly, the median OS of 24 months in the PD-L1 positive NIVO1/ IPI3 cohort is impressive. This is in concert with the results of CheckMate 275 (7), which is reassuring. Nevertheless, the predictive value of this marker cannot be assessed by this study, because of the lack of a non-immunotherapy arm, the retrospective nature of PD-L1 assessment and the small size of the subgroups analyzed. It is also interesting that the cohorts of patients who had PD-L1 expression assessed in their tumors seemed to have a (numerically at least) better OS. The authors do not report the OS according to the assessment of PD-L1 or not, but if this observation is true, it might implicate a bias in this analysis.

In conclusion, Checkmate 032 is the first study to report promising efficacy of nivolumab and ipilimumab combination for previously treated mUC. Despite initial enthusiasm, results should be interpreted with caution. Ongoing phase III trials are eagerly awaited to clarify the role of 1st-line immunotherapy combinations. On the other hand, a better understanding of the mechanisms implicated in response to immune-based therapies may lead to the discovery of novel biomarkers and allow physicians to identify patients likely to benefit from these therapies.

\section{Acknowledgments}

Funding: None.

\section{Footnote}

Conflicts of Interest: All authors have completed the ICMJE uniform disclosure form (available at http://dx.doi. org/10.21037/cco.2019.10.07). The authors have no conflicts of interest to declare.

Ethical Statement: The authors are accountable for all aspects of the work in ensuring that questions related to the accuracy or integrity of any part of the work are appropriately investigated and resolved.

Open Access Statement: This is an Open Access article 
distributed in accordance with the Creative Commons Attribution-NonCommercial-NoDerivs 4.0 International License (CC BY-NC-ND 4.0), which permits the noncommercial replication and distribution of the article with the strict proviso that no changes or edits are made and the original work is properly cited (including links to both the formal publication through the relevant DOI and the license). See: https://creativecommons.org/licenses/by-nc-nd/4.0/.

\section{References}

1. Bamias A, Tiliakos I, Karali MD, et al. Systemic chemotherapy in inoperable or metastatic bladder cancer. Ann Oncol 2006;17:553-61.

2. Bamias A, Tzannis K, Harshman LC, et al. Impact of contemporary patterns of chemotherapy utilization on survival in patients with advanced cancer of the urinary tract: a Retrospective International Study of Invasive/ Advanced Cancer of the Urothelium (RISC). Ann Oncol 2018;29:361-9.

3. Bellmunt J, de Wit R, Vaughn DJ, et al. Pembrolizumab as Second-Line Therapy for Advanced Urothelial Carcinoma. N Engl J Med 2017;376:1015-26.

4. Powles T, Durán I, van der Heijden MS, et al. Atezolizumab versus chemotherapy in patients with platinum-treated locally advanced or metastatic urothelial carcinoma (IMvigor211): a multicentre, open-label, phase 3 randomised controlled trial. Lancet 2018;391:748-57.

5. Balar AV, Galsky MD, Rosenberg JE, et al. Atezolizumab as first-line treatment in cisplatin-ineligible patients with locally advanced and metastatic urothelial carcinoma: a single-arm, multicentre, phase 2 trial. Lancet 2017;389:67-76.

6. Massard C, Gordon MS, Sharma S, et al. Safety and Efficacy of Durvalumab (MEDI4736), an AntiProgrammed Cell Death Ligand-1 Immune Checkpoint Inhibitor, in Patients With Advanced Urothelial Bladder Cancer. J Clin Oncol 2016;34:3119-25.

7. Sharma P, Retz M, Siefker-Radtke A, et al. Nivolumab in metastatic urothelial carcinoma after platinum therapy (CheckMate 275): a multicentre, single-arm, phase 2 trial. Lancet Oncol 2017;18:312-22.

8. Patel MR, Ellerton J, Infante JR, et al. Avelumab in metastatic urothelial carcinoma after platinum failure (JAVELIN Solid Tumor): pooled results from two expansion cohorts of an open-label, phase 1 trial. Lancet Oncol 2018;19:51-64.
9. Sharma P, Siefker-Radtke A, de Braud F, et al. Nivolumab Alone and With Ipilimumab in Previously Treated Metastatic Urothelial Carcinoma: CheckMate 032 Nivolumab $1 \mathrm{mg} / \mathrm{kg}$ Plus Ipilimumab $3 \mathrm{mg} / \mathrm{kg}$ Expansion Cohort Results. J Clin Oncol 2019;37:1608-16.

10. Bamias A, Hegele A, Medioni J, et al. Vinflunine in the treatment of relapsed metastatic urothelial cancer: A systematic review and meta-analysis of real-world series. Crit Rev Oncol Hematol 2019;140:80-7.

11. Bellmunt J, Choueiri TK, Fougeray R, et al. Prognostic factors in patients with advanced transitional cell carcinoma of the urothelial tract experiencing treatment failure with platinum-containing regimens. J Clin Oncol 2010;28:1850-5.

12. Wolchok JD, Chiarion-Sileni V, Gonzalez R, et al. Overall Survival with Combined Nivolumab and Ipilimumab in Advanced Melanoma. N Engl J Med 2017;377:1345-56.

13. Hussain SA, Birtle A, Crabb S, et al. From Clinical Trials to Real-life Clinical Practice: The Role of Immunotherapy with PD-1/PD-L1 Inhibitors in Advanced Urothelial Carcinoma. Eur Urol Oncol 2018;1:486-500.

14. Swartz MA, Iida N, Roberts EW, et al. Tumor microenvironment complexity: emerging roles in cancer therapy. Cancer Res 2012;72:2473-80.

15. D'Errico G, Machado HL, Sainz B, Jr. A current perspective on cancer immune therapy: step-by-step approach to constructing the magic bullet. Clin Transl Med 2017;6:3.

16. Motzer RJ, Tannir NM, McDermott DF, et al. Nivolumab plus Ipilimumab versus Sunitinib in Advanced Renal-Cell Carcinoma. N Engl J Med 2018;378:1277-90.

17. Wolchok JD, Rollin L, Larkin J. Nivolumab and Ipilimumab in Advanced Melanoma. N Engl J Med 2017;377:2503-4.

18. Antonia SJ, Lopez-Martin JA, Bendell J, et al. Nivolumab alone and nivolumab plus ipilimumab in recurrent smallcell lung cancer (CheckMate 032): a multicentre, openlabel, phase 1/2 trial. Lancet Oncol 2016;17:883-95.

19. Hellmann MD, Ciuleanu TE, Pluzanski A, et al. Nivolumab plus Ipilimumab in Lung Cancer with a High Tumor Mutational Burden. N Engl J Med 2018;378:2093-104.

Cite this article as: Economopoulou P, Bamias A. Combination immunotherapy in metastatic urothelial cancer: time for the next step? Chin Clin Oncol 2020;9(2):23. doi: 10.21037/cco.2019.10.07 